\title{
Satisfacción laboral en profesionales de enfermería de un instituto especializado de Perú
}

Job satisfaction in nursing professionals of a specialized institute in Peru

\author{
Gael Campos Truyenque ${ }^{1,2, a}$, Violeta Alicia Nolberto Sifuentes ${ }^{2, b}$, Daysi Milsa Coras Bendezú 3,a,d
}

\section{RESUMEN}

Objetivo: Determinar la satisfacción laboral de las profesionales de enfermería, considerando, dimensiones como el significado de su trabajo, las condiciones laborales, el reconocimiento personal y/o social y los beneficios económicos, además de características generales de la población. Material y métodos: Estudio de tipo descriptivo, de diseño no experimental, cuya población de estudio fue 112 enfermeras que laboran en el Instituto Nacional de Ciencias Neurológicas de Lima-Perú. El censo se realizó a 112 enfermeras, a quienes se les aplicó una escala validada de satisfacción laboral. Se utilizó estadística descriptiva. Resultados: Las enfermeras del estudio, tienen un promedio de edad de 41 años, $86 \%$ son mujeres, $50 \%$ casadas y $50 \%$ laboran entre 6 a 15 años en el instituto. El $48 \%$ de enfermeras presentó satisfacción promedio y $49 \%$ están insatisfechas laboralmente; además el 51\% presentó insatisfacción laboral en las dimensiones Reconocimiento personal y/o social y 58\% en la dimensión Condiciones de Trabajo, respectivamente. Conclusiones: La satisfacción laboral de las enfermeras se encuentra de un nivel promedio a insatisfacción, observándose más insatisfacción en la dimensión Condiciones de Trabajo, por lo que se sugiere implementar propuestas de mejora de las condiciones del colectivo de enfermería con la finalidad de garantizar la calidad de cuidado que brindan a los usuarios de dicho instituto.

PALABRAS CLAVE: Satisfacción en el trabajo, enfermeros.

\section{SUMMARY}

Objective: To determine the job satisfaction of nursing professionals, considering, dimensions such as the meaning of their work, working conditions, personal social recognition and economic benefits, as well as general characteristics of the population. Methodology: Descriptive research, non-experimental design, whose study population was 112 nurses who work in the National Institute of Neurological Sciences. The data collection was made based on a complete census. A standardized questionnaire was applied, which contained sociodemographic questions and a job satisfaction scale. Descriptive statistics was used. Results: The majority of nurses have an average age of 41 years, women (86\%), married (50\%), work between 6 to 15 years in the institute, $(50 \%)$. Nurses presented an average satisfaction (48\%) and they are dissatisfied with their work (49\%). In addition, nurses presented work dissatisfaction in the dimensions of Personal and / or Social Recognition (51\%) and Working Conditions dimension (58\%), respectively. Conclusions: The job satisfaction of nurses is from an average level to a level of dissatisfaction, with more dissatisfaction observed in the Working Conditions dimension, being necessary to improve the elements that allow the activity of the nurse.

KEYWORDS: Job satisfaction, nurses.

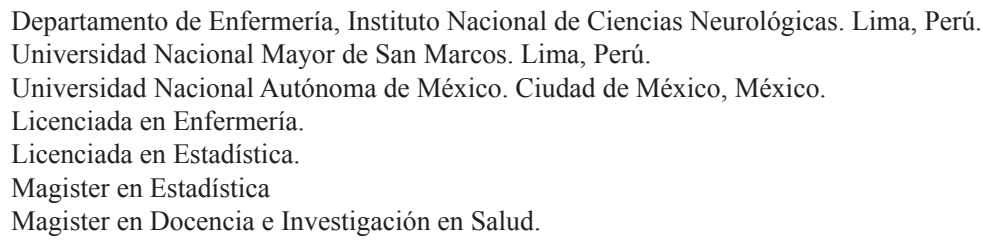




\section{INTRODUCCIÓN}

Uno de los aspectos que ha recibido mayor atención en los ambientes de trabajo es la satisfacción laboral, ya que el recurso humano es importante para que una institución obtenga resultados positivos en términos de productividad, eficiencia e indicadores adecuados de atención de salud, pues la satisfacción favorable o desfavorable del personal influirá en la relación que este adopte con la institución (1). Es así, que la satisfacción laboral del profesional de la salud influye directamente en la calidad de los servicios de salud, donde incluso algunos estudios han considerado que el aumento de la satisfacción laboral del profesional optimiza la relación con el paciente, y con ello se incrementa la calidad de los cuidados prestados, además que la satisfacción laboral influye en la actitud del trabajador y en su desenvolvimiento (2).

Una de las definiciones más clásicas de la satisfacción laboral es dada por Locke, quien la define como "un estado emocional positivo y placentero resultante de la valoración personal que hace el individuo sobre su trabajo y sobre la experiencia adquirida en el mismo" (3)con tamaño de Alfa de Cronbach entre 0.7 y 0.9. Los valores de satisfacción laboral están alrededor de 4 (escala de 1 a 5 . Es así como la idea de satisfacción nace de la comparación entre el trabajo real y las expectativas que el trabajador tiene respecto que "A mayor satisfacción laboral, mayor compromiso del trabajador en el desempeño de sus tareas; por el contrario, si la satisfacción en el trabajo es escasa o nula, no pondrá demasiado empeño en su actividad diaria" (2).

Así mismo, cabe destacar que en algunos estudios realizados en países como México y España, las enfermeras se encuentran medianamente satisfechas (4-6) tal situación muestra la realidad laboral que vive la profesión. Es conocido que el trabajo de enfermería debe reunir las condiciones necesarias y suficientes para que se brinde el cuidado humanizado, dirigida hacia el bienestar de los demás, con un compromiso subyacente de valores y moralidad para cuidar y el anhelo de hacerlo (7).

En tal sentido, la eficiencia de los servicios de enfermería en los hospitales depende también de la gestión de la enfermera jefe, quien debe desempeñarse como líder y administradora para dirigir las actividades de gestión de su competencia. Así, su liderazgo determinará los resultados de su personal, pues su labor, es hacer cumplir las funciones asignadas desde una perspectiva estratégica, a fin de desarrollar el potencial de cada enfermera con liderazgo asertivo.

Por su parte, el Instituto de Nacional de Ciencias Neurológicas (INCN) tiene como misión, la prestación de servicios de salud con calidad a sus usuarios, estando inherente a la función que cumple el Departamento de Enfermería, en el cuidado a los pacientes. Es por ello, que en la actualidad, hay preocupación por ir mejorando el entorno laboral de las enfermeras, para aumentar su satisfacción laboral, lograr los objetivos institucionales y superar las exigencias del trabajo.

Si bien empíricamente se percibe cierta insatisfacción de las enfermeras en el INCN, según expresiones de quejas constantes sobre diversos aspectos laborales relacionados con las remuneraciones, el entorno físico, los jefes, los compañeros de trabajo, el exceso de trabajo y el poco valor que da la institución respecto al tema. Aún, en el INCN, no se tiene información actual sobre la satisfacción laboral que las enfermeras tienen frente a su desempeño, lo que hace necesario identificar esta variable desde varios aspectos, y encontrar sus posibles asociaciones con algunas características sociodemográficas; pues los equipos de enfermería más exitosos buscan lograr que el personal se sienta involucrado con su trabajo.

El objetivo del presente trabajo fue determinar la satisfacción laboral de las profesionales de enfermería, considerando, dimensiones como el significado de su trabajo, las condiciones laborales, el reconocimiento personal y/o social y los beneficios económicos, además de características generales de la población.

\section{MATERIAL Y MÉTODOS}

Se realizó un estudio descriptivo, transversal, no experimental, durante tres meses. De una población total de 112 enfermeras del INCN de la ciudad de Lima- Perú, 105 enfermeras participaron en el estudio correspondiendo a una tasa de respuesta de 93,7\%. Se consideró como criterios de inclusión enfermeras que laboran más de un año en el instituto y que hayan aceptado participar voluntariamente en el estudio; y como criterios de exclusión enfermeras que estuvieran de descanso médico, vacaciones o licencia. La recolección de información se realizó en base a un censo completo. Se aplicó una escala de satisfacción laboral desarrollada y validada por Sonia Palma (8), tal instrumento fue sometido a una prueba piloto a fin de determinar su confiabilidad, obteniendo como 
resultado un coeficiente de Alfa de Cronbach de 0.90, siendo el instrumento confiable. Este instrumento consta de 27 ítems, de los cuales las preguntas 2, 5, $6,10,11,12,13,17,19,24$ son de tipo negativa. La escala se agrupa en 4 dimensiones: Significación de la Tarea, Condiciones de Trabajo, Reconocimiento Personal y/o Social, y Beneficios Económicos (8).

Para la recopilación de la información se contactó a las enfermeras y solicitó su participación voluntaria a través del consentimiento informado de manera presencial y por correo electrónico. A aquellas enfermeras que aceptaron participar en desarrollar el cuestionario, se les entregó de manera física el cuestionario en el establecimiento de salud; y además, se respetó la decisión de las enfermeras que no desearon participar en la investigación. Para el procesamiento de la información, los cuestionarios recibidos fueron digitalizados en hoja de cálculo de Microsoft Excel 2017. Luego fueron procesados y analizados con el software SPSS (versión 23). Se realizó estadística descriptiva para el análisis de datos y se presentaron los resultados en tablas de frecuencias y porcentajes.

El presente estudio cuenta con la autorización del Departamento de Enfermería del INCN y el Comité de Ética de la Universidad Nacional Mayor de San Marcos.

\section{RESULTADOS}

En la tabla 1 se aprecian las características generales de las enfermeras, donde el promedio de edad es 41 años con una DS $\pm 7,5$. La población de estudio es de predominio del sexo femenino (86\%); además son casadas (50\%); que laboran entre 6 a 15 años en la institución (50\%), cuya condición laboral es de nombramiento $(86 \%)$, mientras que un grupo reducido tiene estudios de post grado en maestría o doctorado $(22 \%)$.

Tabla 1. Características generales de las enfermeras del Instituto Nacional de Ciencias Neurológicas.

\begin{tabular}{|c|c|c|}
\hline Características Generales & $\mathrm{n}$ & $\%$ \\
\hline Edad (media \pm DS) & $\begin{array}{c}41 \pm \\
7,5\end{array}$ & \\
\hline \multicolumn{3}{|l|}{ Sexo } \\
\hline Femenino & 90 & 86 \\
\hline Masculino & 15 & 14 \\
\hline \multicolumn{3}{|l|}{ Estado Civil } \\
\hline Soltera & 51 & 49 \\
\hline Casada & 53 & 50 \\
\hline Separada & 1 & 1 \\
\hline \multicolumn{3}{|l|}{ Tiempo de servicio } \\
\hline Menos de 5 años & 12 & 11 \\
\hline 6 a 15 años & 52 & 50 \\
\hline 16 a 25 años & 26 & 25 \\
\hline Mas de 25 años & 15 & 14 \\
\hline \multicolumn{3}{|l|}{ Condición Laboral } \\
\hline Nombramiento & 90 & 86 \\
\hline Contrato & 15 & 14 \\
\hline \multicolumn{3}{|l|}{ Estudios de Especialidad } \\
\hline $\mathrm{Si}$ & 103 & 98 \\
\hline No & 2 & 2 \\
\hline \multicolumn{3}{|l|}{ Estudios de Maestría y doctorado } \\
\hline $\mathrm{Si}$ & 23 & 22 \\
\hline No & 82 & 78 \\
\hline
\end{tabular}


Tabla 2. Satisfacción laboral y dimensiones, en el profesional de enfermería del Instituto Nacional de Ciencias Neurológicas.

\begin{tabular}{|c|c|c|c|c|c|c|c|c|c|}
\hline \multirow{3}{*}{ Nivel } & \multirow{2}{*}{\multicolumn{2}{|c|}{$\begin{array}{c}\text { Satisfacción } \\
\text { Laboral Global }\end{array}$}} & \multicolumn{7}{|c|}{ Dimensiones de la Satisfacción Laboral } \\
\hline & & & \multicolumn{2}{|c|}{$\begin{array}{l}\text { Significación de } \\
\text { la tarea }\end{array}$} & \multicolumn{2}{|c|}{$\begin{array}{c}\text { Condiciones de } \\
\text { trabajo }\end{array}$} & \multicolumn{2}{|c|}{$\begin{array}{c}\text { Reconocimiento } \\
\text { personal y/o social }\end{array}$} & $\begin{array}{l}\text { Beneficios } \\
\text { Económicos }\end{array}$ \\
\hline & $\mathrm{n}$ & $\%$ & $\mathrm{n}$ & $\%$ & $\mathrm{n}$ & $\%$ & $\mathrm{n}$ & $\%$ & $\mathrm{n} \%$ \\
\hline Satisfecho & 3 & 3 & 45 & 43 & 2 & 2 & 21 & 20 & 2322 \\
\hline Promedio & 50 & 48 & 50 & 48 & 42 & 40 & 30 & 29 & 5956 \\
\hline Insatisfecho & 52 & 50 & 10 & 10 & 61 & 58 & 54 & 52 & $23 \quad 22$ \\
\hline
\end{tabular}

Tabla 3. Descripción de la satisfacción laboral en el profesional de enfermería del Instituto Nacional de Ciencias Neurológicas.

\begin{tabular}{|c|c|c|c|c|}
\hline \multirow[t]{2}{*}{ Ítems $(n=105)$} & $\begin{array}{c}\text { De } \\
\text { acuerdo }\end{array}$ & Indeciso & \\
\hline & n $\%$ & n $\%$ & $\mathbf{n}$ & $\%$ \\
\hline $\begin{array}{l}\text { 1. La distribución física del ambiente de trabajo facilita la realización de } \\
\text { mis labores. }\end{array}$ & 2322 & 2221 & 60 & 57 \\
\hline 2. Mi sueldo es muy bajo para la labor que realizo. & $87 \quad 83$ & 1110 & 7 & 7 \\
\hline 3. Siento que el trabajo que hago es justo para mi manera de ser. & 3432 & $38 \quad 36$ & 33 & 31 \\
\hline 4. La tarea que realizo es tan valiosa como cualquier otra. & 9288 & $8 \quad 8$ & 5 & 5 \\
\hline 5. Me siento mal con lo que gano. & $67 \quad 64$ & 2322 & 15 & 14 \\
\hline 6. Siento que recibo "mal trato" de parte de la institución. & 1918 & 5451 & 32 & 30 \\
\hline 7. Me siento útil con la labor que realizo. & 9691 & 55 & 4 & 4 \\
\hline 8. El ambiente donde trabajo es confortable. & 55 & 2322 & 77 & 73 \\
\hline 9. El sueldo que tengo es bastante aceptable. & 2221 & $28 \quad 27$ & 55 & 52 \\
\hline 10. La sensación que tengo de mi trabajo es que me están explotando. & 2120 & 4644 & 38 & 36 \\
\hline 11. Prefiero tomar distancia con las personas con quienes trabajo. & $15 \quad 14$ & 2524 & 65 & 62 \\
\hline 12. Me disgusta mi horario. & 4947 & 3432 & 22 & 21 \\
\hline 13. Las tareas que realizo las percibo como algo sin importancia. & 2928 & 5149 & 25 & 24 \\
\hline 14. Llevarse bien con el jefe beneficia la calidad del trabajo. & 2120 & $65 \quad 62$ & 19 & 18 \\
\hline 15. La comodidad del ambiente de trabajo es inigualable. & 66 & 1211 & 87 & 83 \\
\hline 16. Mi trabajo me permite cubrir mis expectativas económicas. & 1110 & 22 & 92 & 88 \\
\hline 17. El horario de trabajo me resulta incómodo. & 7470 & 1615 & 15 & 14 \\
\hline 18. Me complace los resultados de mi trabajo. & $68 \quad 65$ & 2221 & 15 & 14 \\
\hline 19. Compartir el trabajo con otros compañeros me resulta aburrido. & $15 \quad 14$ & 1918 & 71 & 68 \\
\hline 20. En el ambiente físico en el que laboro me siento cómodo. & $25 \quad 24$ & 2221 & 58 & 55 \\
\hline 21. Mi trabajo me hace sentir realizado como persona. & 8884 & 1010 & 7 & 7 \\
\hline 22. Me gusta el trabajo que realizo. & 8278 & 1110 & 12 & 11 \\
\hline 23. Existen las comodidades para un buen desempeño de las labores diarias. & 44 & $5 \quad 5$ & 96 & 91 \\
\hline 24. Me desagrada que limiten mi trabajo para no reconocer las horas extras. & 3634 & 3634 & 34 & 31 \\
\hline 25. Haciendo mi trabajo me siento bien conmigo mismo. & 9893 & $4 \quad 4$ & 3 & 3 \\
\hline 26. Me gusta la actividad que realizo. & 9994 & 55 & 1 & 1 \\
\hline 27. Mi jefe valora el esfuerzo que pongo en mi trabajo. & $48 \quad 46$ & 2322 & 34 & 32 \\
\hline
\end{tabular}


En la tabla 2 se observa que la mayoría de las enfermeras presenta insatisfacción laboral (50\%), seguido de satisfacción laboral promedio (48\%). A nivel de las dimensiones de la Satisfacción Laboral, se evidencia que en la dimensión Significación de la Tarea predomina una satisfacción promedio (48\%), en la dimensión Condiciones de Trabajo, la mayoría de las enfermeras se sienten insatisfechas (58\%), así también se observa un alto porcentaje de insatisfacción en la dimensión Reconocimiento personal y/o social (52\%), mientras que en la dimensión Beneficios económicos se observa una satisfacción a nivel promedio $(56 \%)$.

La tabla 3 muestra los porcentajes de concordancia por cada ítem de la Escala de Satisfacción Laboral. Específicamente las enfermeras refieren que gustan de la actividad que realizan en alto nivel de acuerdo (94\%), que al hacer su trabajo se sienten bien consigo mismas $(93 \%)$, su trabajo las hace sentir realizadas como persona $(84 \%)$ y se sienten útiles con la labor que realizan (91\%), mientras que existe un desacuerdo muy evidente respecto a que el trabajo les permita cubrir sus expectativas económicas $(88 \%)$, que la comodidad del ambiente de trabajo es inigualable $(83 \%)$ y que el sueldo que tienen sea bastante aceptable (52\%). Así mismo, este instrumento muestra ítems negativos; destacando que el compartir el trabajo con otros compañeros les resulta aburrido (68\%), mientras que algunos ítems negativos tienen un alto nivel de acuerdo y aceptación de las enfermeras tales como, que su sueldo es muy bajo para la labor que realizan (83\%) y que se sienten mal con lo que ganan (64\%). También se observa que las enfermeras están indecisas respecto a que reciban mal trato de parte de la institución (51\%) y que las tareas que realizan sean algo sin importancia $(49 \%)$.

\section{DISCUSIÓN}

El presente estudio permitió identificar la Satisfacción Laboral de las enfermeras del INCN, donde se pudo evidenciar una proporción similar entre satisfacción e insatisfacción, esta realidad es distinta, a lo obtenido por Bobbio y Ramos en un estudio peruano, donde la satisfacción laboral del personal no médico (enfermeras y obstetrices) alcanza el 26\% y la insatisfacción el 74\% (9), y de igual forma en el estudio de Carrillo et al., donde las enfermeras destacaron por ser las que ofrecen niveles de satisfacción más reducidos (10), mientras que en otros estudios $(4,5)$ en México reportan, que las enfermeras están medianamente satisfechas. Similar situación se observa en España en el estudio de Pablos \& Cubo donde la satisfacción en el grupo de enfermeras de tres hospitales es de nivel medio a alto (6). Este panorama nos muestra que existen diferencias entre la satisfacción laboral de enfermeras peruanas, con la satisfacción laboral de enfermeras de otros países, resaltando así un gran porcentaje de insatisfacción en el presente estudio, siendo dado probablemente por factores institucionales y también por el propio sistema de salud de nuestro país, que evidencia deficiencias y problemas que tanto los enfermeros y otros profesionales y trabajadores las comparten. El actual Sistema de Salud afronta un presupuesto insuficiente para el sector, en dotación deficiente de recursos humanos (médicos, enfermeras, personal técnico, etc.) en todos los hospitales del país; y la modernización de los hospitales tanto en infraestructura y equipos se encuentran en proceso. Los esfuerzos técnicos para modernizar el sistema de salud, es resaltable sin embargo necesita mayor celeridad (11).

Sobre la Insatisfacción Laboral, Carrillo et al., considera que los profesionales de enfermería son los que se encuentran en más contacto con el paciente, y es en las enfermeras en quienes recae la responsabilidad de viabilizar o solucionar problemas o dilemas que surgen durante la práctica diaria, además de la sobrecarga laboral, relaciones interpersonales deficientes, falta de incentivos, entre otros factores de carácter externo pudieran explicar la insatisfacción con el trabajo (10), y tal como lo sustentan Cifuentes y Manrique se pudiera llevar también al ausentismo y al abandono del trabajo, siendo la insatisfacción laboral una desventaja para toda organización (12).

Esta investigación también identificó la satisfacción laboral en las enfermeras del INCN en cuatro dimensiones, siendo: Significación de la Tarea, Condiciones de Trabajo, Reconocimiento personal y/o social y Beneficios Económicos. Respecto a la dimensión Significación de la Tarea, que define como una valoración que la persona tiene hacia su trabajo, teniendo en cuenta el sentido de esfuerzo del trabajador, la realización con su trabajo, el sentimiento de equidad y el aporte material que puede tener, en el estudio se observa que existe satisfacción promedio en esta dimensión y solo pocas enfermeras se consideran insatisfechas; esta situación es similar a lo reportado por Juárez quien en su investigación, el $45 \%$ de enfermeras se siente satisfecha con las oportunidades de desarrollo en su trabajo, indicador que es semejante a la definición de la dimensión de Significación de la Tarea en el estudio (5), así mismo, el autor registró que el $70 \%$ de enfermeras en su estudio se encuentran 
satisfechas con la forma en cómo se realiza su trabajo, siendo también distinto con el presente estudio ya que en la dimensión significación de la tarea se registra una satisfacción media o regular.

En la dimensión Condiciones de Trabajo, que se define como la disposición de los elementos o materiales o normas que permiten la actividad laboral del trabajador, en el estudio se encontró que la mayoría de enfermeras esta insatisfecha, mientras que Juárez reporta una mediana satisfacción respecto al ambiente físico de trabajo (4) y de igual forma Carrillo et al., manifiestan que las enfermeras en su estudio tienen satisfacción media respecto a las condiciones físicas del trabajo (13), tales situaciones evidencian que existe una común calificación a como las enfermeras valoran las condiciones en las que trabajan, donde es necesario intervenir, pues tales deficiencias tienen consecuencias en la atención del paciente y en la propia carga de trabajo de la enfermera.

En relación a la dimensión Reconocimiento personal $y /$ o social, que se define como el reconocimiento propio o de las personas que se encuentran vinculadas con la supervisión del trabajo de la persona, valorando los logros en el trabajo; en el estudio se observa que casi la mitad de las enfermeras del instituto esta insatisfecha, situación similar al estudio de Carrillo et al., donde se obtienen niveles de satisfacción negativos respecto con las posibilidades de promoción o ascenso (10), además en el estudio de Carrillo et al., las enfermeras se sienten medianamente satisfechas respecto al reconocimiento que obtienen por el trabajo bien hecho (13). Probablemente esta situación se evidencie, pues es común observar que las posibilidades de reconocimiento en el trabajo se dan por factores como antigüedad o cercanía a la gestión jefatural, mas no por factores que tomen en cuenta los méritos personales o profesionales, los cuales conllevan a insatisfacciones y desmotivaciones. Tal situación también es señalada por Gillet et al., quien manifiesta en su estudio, que la satisfacción laboral se relaciona con el apoyo percibido de parte del supervisor de enfermería (15), lo que confirma la importancia de que las autoridades de enfermería manejen ciertas aptitudes de liderazgo asertivo, que favorezca al crecimiento y la satisfacción de las enfermeras.

En la dimensión Beneficios Económicos, que trata sobre los incentivos o remuneraciones que recibe el trabajador, en el estudio se observa satisfacción promedio, lo cual es similar al estudio realizado por Bobbio y Ramos donde el 31\% estaba satisfecho con su salario, y el 15\% satisfecho con los incentivos económicos (9), mientras que Carrillo et al., reportaron insatisfacción de las enfermeras con respecto al salario (13); y de igual forma lo evidenció el estudio de Andrioti et al., (16). La realidad de las remuneraciones adecuadas al personal asistencial de salud, debería tomarse más en cuenta, ya que son las enfermeras, personal técnico de enfermería y también médicos quienes se encuentran expuestos a una serie de enfermedades dentro de los hospitales, además de trabajar en horarios nocturnos, domingos y feriados, lejos de la familia y seres queridos, sin embargo, se observa que el Estado Peruano otorga menor presupuesto al sector de salud a comparación de otros sectores, siendo así las remuneraciones bajas o muy básicas, y esta austeridad económica en el sector, provoca también racionalización de materiales y recursos en los hospitales, los cuales influyen en brindar una atención de calidad o no a los pacientes.

El presente estudio tiene ciertas limitaciones ya que solo se dirigió la investigación a enfermeras de una institución pública de la ciudad de Lima, por lo que los resultados no pueden generalizarse a todas las enfermeras a nivel regional o nacional. Así mismo, la información obtenida proviene de una escala, respondida de manera anónima por las enfermeras, por lo que las respuestas están limitadas a la sinceridad de las participantes.

La información de esta investigación a nivel institucional y de otras organizaciones de salud, permite proponer estrategias para el mejoramiento del entorno laboral y la satisfacción de los trabajadores, ya que tanto enfermeras como otros profesionales de la salud, cumplen un rol importante para el logro de los objetivos misionales de una institución de salud.

\section{CONCLUSIONES}

La satisfacción laboral de las enfermeras se encuentra de un nivel promedio a un nivel de insatisfacción, observándose más insatisfacción en la dimensión Condiciones de Trabajo, siendo necesario mejorar los elementos que permitan brindar una mejor atención al paciente neurológico.

Fuentes de financiación: La investigación fue autofinanciada.

Conflictos de interés: El estudio no tuvo ningún conflicto de interés. 


\section{Correspondencia:}

Gael Campos Truyenque

Correo electrónico: gaelcampos26@gmail.com_

\section{REFERENCIAS BIBLIOGRAFICAS}

1. Manosalvas C, Manosalvas L, Nieves J. El clima organizacional y la satisfacción laboral: un análisis cuantitativo riguroso de su relación. AD-minister. 2015;(26):5-15.

2. Sánchez M, Sánchez P. Factores determinantes de la satisfacción laboral en España antes y durante la crisis de 2008. Intang Cap. 2016 ;12(5): 1192-1220.

3. Chiang M, Gómez N, Salazar C. Satisfacción laboral y estilos de liderazgo en instituciones públicas y privadas de educación en Chile. Cuad Adm la Univ del Val. 2013 ;30(52):65-74. (Fecha de acceso 10 de noviembre del 2017) Disponible en: http://www. scielo.org.co/pdf/cuadm/v30n52/v30n52a07.pdf

4. Juarez L. Satisfacción laboral del personal operativo de enfermería y clima organizacional en un hospital de $2^{\circ}$ nivel en Aguascalientes. Tesis de Maestria. San Luis Potosí, Mexico: Universidad Autónoma de San Luis Potosí; 2011. (Fecha de acceso 10 de noviembre del 2017) Disponible en: http://ninive. uaslp.mx/jspui/bitstream/i/3033/4/MAE1SLP01101. pdf

5. Juárez S. Clima organizacional y satisfacción laboral. Rev Médica del Inst Mex Segur Soc. 2012;50(3):30714. (Fecha de acceso 10 de noviembre del 2017) Disponible en: http://www.redalyc.org/ pdf $/ 4577 / 457745495014 . p d f$

6. Pablos M, Cubo S. Satisfacción laboral de las enfermeras de los Hospitales Públicos de Badajoz. Med Segur Trab (Madr). 2015; 61(239):195-206. (Fecha de acceso 10 de noviembre del 2017) Disponibleen:http://scielo.isciii.es/scielo.php?script= sci_arttext\&pid=S0465-546X2015000200006\&lng= en\&nrm=iso\&tlng=en

7. Fernández B, Paravic T. Nivel de Satisfacción laboral en enfermeras de hospitales públicos y privados de la provincia de Concepción Chile. Cienc y enfermería. 2003;9(2):57-66. (Fecha de acceso 10 de noviembre del 2017) Disponible en: http://www. scielo.cl/scielo.php?script=sci arttext\&pid=S0717$95532003000200006 \& \operatorname{lng}=\mathrm{en} \& \mathrm{nrm}=\mathrm{iso} \& \operatorname{lng}=\mathrm{en}$
8. Palma S. Elaboración y validación de una escala de satisfacción laboral SL-SPC para trabajadores de Lima Metropolitana. Buenos Aires: El Cid Editor; 2005.

9. Bobbio L, Ramos W. Satisfacción laboral y factores asociados en personal asistencial médico y no médico de un hospital nacional de Lima-Perú. Rev Peru Epidemiol. 2010;14(2):133-8.

10. Carrillo C, Martínez M, Gómez C, Meseguer M. Satisfacción laboral de los profesionales sanitarios de un Hospital Universitario: análisis general y categorías laborales. An Psicol. 2015; 31(2):645. DOI: https://doi.org/10.6018/analesps.31.2.169791

11. Sánchez F. El Sistema Nacional de Salud del Perú. Rev Peru Med Exp Salud Publica. 2014;31(4):747-53. (Fecha de acceso 10 denoviembre del2017)Disponible en: http://www.scielo.org.pe/scielo.php?script=sci_ar ttext\&pid=S1726-46342014000400021

12. Cifuentes J, Manrique F. Satisfacción laboral en enfermería en una institución de salud de cuarto nivel de atención, Bogotá, Colombia. Av en Enfermería. 2014;32(2): 217-27. (Fecha de acceso 10 de noviembre del 2017) Disponible en: http:// www.scielo.org.co/pdf/aven/v32n2/v32n2a05.pdf

13. Carrillo-Garcia C, Ríos-Risquez $M$, FernándezCánovas M, Celdrán-Gil F, Vivo-Molina M, MartínezRoche M. La satisfacción de la vida laboral de los enfermeros de las unidades móviles de emergencias de la Región de Murcia. Enfermería Glob. 2015; 14(40): 266-75. DOI: https://doi.org/10.6018/ eglobal.14.4.198061

14. Carrillo C, Martínez M, Gómez C, Meseguer M. Satisfacción laboral de los profesionales sanitarios de un Hospital Universitario: análisis general y categorías laborales. An Psicol. 2015 ;31(2):645-50. DOI: http://dx.doi.org/10.6018/analesps.31.2.169791

15. Gillet N, Fouquereau E, Coillot H, et al. The effects of work factors on nurses' job satisfaction, quality of care and turnover intentions in oncology. J Adv Nurs. 2018 ; 74(5):1208-19. DOI: http://doi.wiley. com/10.1111/jan. 13524

16. Andrioti D, Skitsou A, Karlsson LE, Pandouris C, Krassias A, Charalambous G. Job Satisfaction of Nurses in Various Clinical Practices. Int J Caring Sci. 2017;10(1):1-76. 